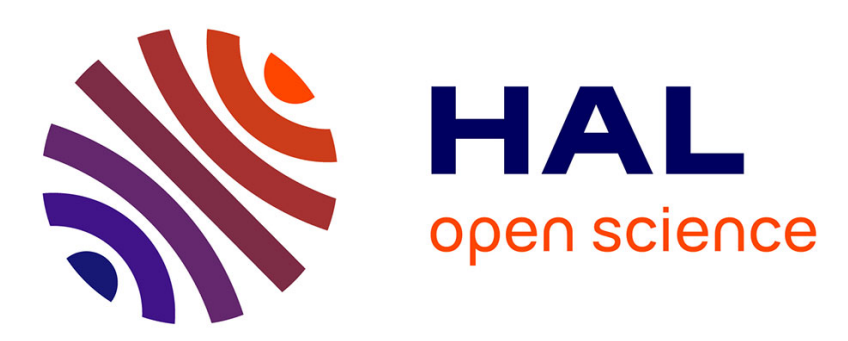

\title{
Quadrupolar solid-state NMR and repetitive experiments: Some aspects in the Liouville space. Application to spins $\mathrm{I}=1$ \\ Christophe Odin
}

\section{- To cite this version:}

Christophe Odin. Quadrupolar solid-state NMR and repetitive experiments: Some aspects in the Liouville space. Application to spins I=1. Solid State Nuclear Magnetic Resonance, 2017, 84, pp.7382. 10.1016/j.ssnmr.2016.12.012 . hal-01810673

HAL Id: hal-01810673 https://hal-univ-rennes1.archives-ouvertes.fr/hal-01810673

Submitted on 8 Jun 2018

HAL is a multi-disciplinary open access archive for the deposit and dissemination of scientific research documents, whether they are published or not. The documents may come from teaching and research institutions in France or abroad, or from public or private research centers.
L'archive ouverte pluridisciplinaire HAL, est destinée au dépôt et à la diffusion de documents scientifiques de niveau recherche, publiés ou non, émanant des établissements d'enseignement et de recherche français ou étrangers, des laboratoires publics ou privés. 


\title{
Quadrupolar solid-state NMR and repetitive experiments : some aspects in the Liouville space. Application to spins $\mathrm{I}=1$.
}

\author{
Christophe ODIN \\ ${ }^{1}$ Institut of Physics of Rennes IPR/UMR CNRS 6251, University of Rennes I, Campus \\ de Beaulieu, Bat 11A, 35042 Rennes Cedex, FRANCE
}

\section{Abstract}

The aim of this work is to generalize the Ernst-Anderson model developed to account of the steady-state regime of isolated spins $I=1 / 2$ subject to a train of strictly identical pulse sequences separated by free evolution periods of same duration. We generalize this model to the general case of spins $I \geq 1$ and general pulse sequence within the framework of the Liouville space. In particular, it is proved that under reasonable assumptions, a well defined steady-state regime is reached which is independent of the initial conditions. The general formal expressions obeyed by the steady-state density operator are given as a function of pulse propagators and relaxation operator for single and two-pulse sequences. In solid-state NMR where recycle time can be made, at the same time, much longer than typical coherence relaxation times and smaller than typical population relaxation times, further simplification leads to more tractable formula. As an example, the formalism is applied to $\mathrm{I}=1$ spins with hard and soft single pulse sequence, or to the solid echo se-

Email address: christophe.odin@univ-rennes1.fr (Christophe ODIN) 
quence. In particular, we were able to generalize the Ernst-Anderson formula to spins $\mathrm{I}=1$. The pertinence of the theory is verified by comparing the theoretical and numerical simulations outputs to ${ }^{2} H$ single crystal experiments performed on nonadecane/urea $C_{19} D_{40} /$ urea $-H_{4}$ compound.

Keywords: Liouville Space, Quadrupolar NMR, Progressive Saturation, Solid-state NMR, Spin I=1, Deuterium

\section{Introduction}

Because of the low sensitivity of NMR, experimentalist have been concerned with the problem to determine the optimum conditions to obtain an improvement in signal to noise ratio. Since most experiments are designed to work with the initial state being the thermodynamical equilibrium state, the co-addition of signals from successive experiments imposes that the recycle time should be at least five times longer than the longest relaxation time of the system. Since the effective Free Induction Decay (FID) acquisition time is of the order of the inverse of a typical linewidth or spectral range to be covered, most of the acquisition time is lost in waiting the system to recover its thermal equilibrium. Therefore, since the beginning of pulsed NMR, the question of whether experiments could be performed with shorter recycle times has been addressed.

When the spin system has not enough time to relax back to its thermodynamical equilibrium, we expect to reach a steady-state dynamical equilibrium that results of the competition between the pulses of the sequence that create and mix-up populations and coherences, and the free evolution periods where populations and coherences relax back to their equilibrium values. The im- 
portance of such a steady-state was soon recognized by H.Y. Carr in 1958 [1]. The theory of progressive saturation experiments developed by Ernst and Anderson [2] for isolated spins $\mathrm{I}=1 / 2$ calculated the effects on the NMR signal of the repetition of identical single pulses separated by a recycle time not long enough to allow the system to relax completely to its thermal equilibrium state. The theory considered the contribution of both longitudinal $T_{1}$ and transversal $T_{2}$ relaxation times $[2,3,4,5]$. If the coherences have not decayed during the recycle time, then the FID could be distorted and additional contributions such as echoes could appear. However, in the case where the coherences have been irreversibly cancelled by some means (for instance with a strong field gradient pulse applied between two RF pulses), it was shown that the longitudinal magnetization attains a well defined steadystate regime of magnetization $M_{s s t}$. For a hard pulse of angle $\beta$, the well known and celebrated Ernst-Anderson formula for $\mathrm{I}=1 / 2$ is

$$
M_{s s t}=\frac{1-e^{-T / T_{1}}}{1-\cos (\beta) e^{-T / T_{1}}} M_{e q}
$$

where $\mathrm{T}$ is the recycle time. Thus, the equilibrium magnetization $M_{e q}$ is just rescaled by a factor which depends on both the recycle time and the pulse angle. Moreover, optimal conditions could be found to enhance the signal to noise ratio per unit time by adjusting the pulse angle. Such a method was also applied to the measurement of spin-lattice relaxation time $T_{1}$ because it is far much less time consuming than the usual inversion- recovery sequence. Moreover, it was shown that the signal $M_{s s t} \sin \beta$ at given recycle time $T$ presents a maximum as a function of pulse length for the Ernst angle

$$
\cos (\beta)=e^{-T / T_{1}}
$$


The theory was also extended to spins $I=1 / 2$ subject to chemical exchange $[6,7]$, showing that large errors on the measurement of $T_{1}$ could be induced by the exchange process. A general formalism in the Liouville space was proposed for steady-state NMR [8].

Mainly, the theory of progressive saturation has only been developed for isolated spins $\mathrm{I}=1 / 2$ where the relaxation of the populations and coherences are each defined by a single relaxation time $T_{1}$ or $T_{2}$. Such results are usually unscrupulously transferred without rigorous justification to spins $I \geq 1$ in order to save experimental time. The idea is that, if $T$ is of the order of the supposed population relaxation times (1 to 3 times ?), dummy scans that consist of the excitation of the sample without recording the signal will lead to a steady-state close to the equilibrium one, especially in solids where population relaxation times are usually much larger than coherence relaxation times. To the best of our knowledge, the case of quadrupolar nuclei was only explicitly treated in [9], but in the special case of only one population relaxation time, with a vector model, and assuming no contribution of the coherences.

The aim of this study is to generalize the Ernst-Anderson model to general pulse sequences and general spin values $I \geq 1 / 2$ within the framework of the Liouville space formalism and reasonable assumptions concerning the treatment of the relaxation (weak collision regime, secular approximation). The case of isolated spins $I \geq 1$ is complicated by the existence of different population relaxation times and numerous coherences relaxation times, as well as by cross relaxation effects. We will show that simple closed formula that generalize the Ernst-Anderson formula can be generalized for spins $\mathrm{I}=1$. 
It is important since deuterium ${ }^{2} H$ NMR is widely used in solid-state and soft-matter NMR [10, 11, 12], as well as ${ }^{14} N$ NMR [13]. Moreover, it is not unusual to find long spin-lattice relaxation times in molecular samples $[14,15,16,17,18,19]$, so any procedures which can save experimental time may have some interest for the experimentalist.

This article is divided as follows. In section 2, we develop a formal solution of the progressive saturation experiment when relaxation is in the Redfield regime, using the formalism of the Liouville space with no restrictions on the value of the spin I and on the type of sequence. Section 3 applies the results to the particular case of an isolated spin $\mathrm{I}=1$, where some simple results similar to the Ernst-Anderson model can be given. The single pulse and solid echo sequences are treated as an example of application. In particular, we will show that a progressive saturation experiment could, in principle, measure the Zeeman order relaxation time. The theory is compared to experimental results in section 4 , obtained from deuterium NMR ${ }^{2} H$ performed on a crystals of nonadecane/urea $C_{19} D_{40} /$ urea $-H_{4}$ with single and two pulses sequences.

One Pulse (PRE) as well as Solid-Echo (SERE) Repetitive Experiments will be considered ${ }^{1}$.

PRE : One pulse Repetitive Experiments

SERE : Solid-Echo Repetitive Experiments 


\section{Theory of repetitive experiments}

This section develops the theory of repetitive experiments, that is the continuous excitation of a system by a NMR pulse sequence with a period given by the recycle time $T$, from an undetermined initial condition to the steady-state.

\subsection{Liouville space}

The system is supposed static (no modulation of the interactions by a mechanical rotation with respect to the magnetic field) and free of chemical exchange. The density operator $\rho(t)$ which describes the state of the system at time $t$ acts on a Hilbert space spanned by a basis of state functions. In the weak collision regime, the density operator evolves according to the Redfield equation $[20,21,22,23]$

$$
\frac{\partial \rho}{\partial t}=-i[H, \rho(t)]+\mathbb{R}\left(\rho(t)-\rho_{e q}\right)
$$

$\mathrm{H}$ is a time independent Hamiltonian and the relaxation super-operator $\mathbb{R}$ drives the system towards its thermal equilibrium value $\rho_{e q}$. Typically, the Redfield regime is valid in the motional narrowing limit where typical correlation times are much smaller than the reciprocal rigid lattice linewidth or in the extreme narrowing limit where the correlation times are much smaller than the reciprocal Larmor angular frequencies.

All the Hamiltonians are expressed in units of angular frequencies $(\hbar=1)$. For free evolution, $H=H_{z}$ where $H_{z}$ is the truncated high field free evolution Hamiltonian. During a pulse, the Hamiltonian is $H=H_{z}+H_{r f}$ with, in the rotating frame representation,

$$
H_{r f}=\omega_{1}\left(\cos \varphi I_{x}+\sin \varphi I_{y}\right)
$$


$\varphi$ and $\omega_{1}$ are the pulse phase and irradiation strength respectively. A pulse is said "hard" when $\left\|H_{z}\right\| \ll\left\|H_{r f}\right\|$ and soft otherwise, which is the general case in solid-state NMR.

In the Liouville space $[21,23,24,25,26,27]$, the rotating frame density operator $\rho$ is represented by a column vector $|\rho\rangle$, called hereafter density vector, whose components are the coefficients of the expansion of the density operator on a complete set of orthonormal operators.

The Redfield equation of evolution of the density operator can be cast into a set of linearly coupled first order equation for the density vector in the Liouville space as

$$
\frac{\partial|\rho\rangle}{\partial t}=-i \mathcal{H}^{l}|\rho(t)\rangle+\mathbb{R}\left(|\rho(t)\rangle-\left|\rho_{e q}\right\rangle\right)
$$

where $\mathcal{H}^{l}$, the Liouville super-operator associated to operator $\mathrm{H}$, is Hermitian. The relaxation super-operator $\mathbb{R}$ is also Hermitian. In the high field approximation, $\mathbb{R}$ commutes with the Liouville super-operator generated by $J_{z}$.

The general solution of Eq. [5] when only free evolution is concerned can be given. Because $\mathcal{H}_{z}^{l}\left|\rho_{\text {eq }}\right\rangle=|0\rangle$, where $|0\rangle$ is the null vector Eq. [5] can be recast in the compact form

$$
\frac{\partial|\rho\rangle}{\partial t}=\mathbb{L}_{z}\left(|\rho(t)\rangle-\left|\rho_{e q}\right\rangle\right)
$$

with $\mathbb{L}_{z}=\left(-i \mathcal{H}_{z}^{l}+\mathbb{R}\right)$.

Within these assumptions, the formal solution of Eq.[6] is

$$
|\rho(t)\rangle=\mathbb{E}_{t}|\rho(0)\rangle+\left(\mathbb{I}-\mathbb{E}_{t}\right)\left|\rho_{e q}\right\rangle \text { with } \mathbb{E}_{t}=e^{\mathbb{L}_{z} t}
$$


where $\mathbb{I}$ is the identity operator. The free evolution propagator operator $\mathbb{E}_{t}$ is non unitary because relaxation is an irreversible process. Note also that the two super-operators $\mathcal{H}_{z}^{l}$ and $\mathbb{R}$ do not commute in general, so that the free evolution propagator is not the product of two exponential super-operators.

We now specialize to an isolated spin I. The Liouville space is spanned by $\operatorname{dim} L=(2 I+1)^{2}$ linearly independent operators. $2 \mathrm{I}+1$ operators are related to the populations, 2(2I) operators to the single quantum coherences $1 \mathrm{QC}$, $2(2 \mathrm{I}-1)$ to the $2 \mathrm{QC} \ldots$ and two to the $(2 \mathrm{I}) \mathrm{QC}$. The principal zero eigenvalue of the super-operators could be removed by suppressing the unit operator from the basis and working in a dimL-1 Liouville space. This is equivalent to work with a density operator of zero trace $(\operatorname{Tr}(\rho)=0)$.

It is convenient to partition the components of the vector $|\rho\rangle$ into components related to the populations and components related to $\mathrm{n} \geq 1$ coherence orders $\left|\rho_{c}\right\rangle$ as

$$
|\rho(t)\rangle=\left(\begin{array}{c}
\left|\rho_{p}(t)\right\rangle \\
\left|\rho_{c}(t)\right\rangle
\end{array}\right)
$$

$\left|\rho_{p}\right\rangle$ is the pure population contribution. $\left|\rho_{p}\right\rangle$ has $2 \mathrm{I}+1$ components and $\left|\rho_{c}\right\rangle$ has $2 \mathrm{I}(2 \mathrm{I}+1)$ components.

The thermal equilibrium density vector $\left|\rho_{e q}\right\rangle$ is such that $\left|\rho_{e q, c}\right\rangle=0$. In very high fields, one can in general neglect all contributions except the Zeeman interaction with the static field, so $\rho_{e q} \sim J_{z}$ with $J_{z}=\sum_{i} I_{i z}$ the total angular momentum operator . By convention, we suppose that the first component of the vector $\left|\rho_{e q}\right\rangle$ corresponds to a projection onto $J_{z}$. Designating by $\omega_{l}$ the Zeeman splitting and by $\omega_{i}$ the anisotropy factor in angular 
frequency units of interaction i, the contribution of other interactions such as the quadrupolar interaction to the equilibrium population in the high temperature limit are roughly in the ratio $\sim \frac{\omega_{i}}{\omega_{l}} \ll 1$, and could easily be incorporated if needed.

The matrix corresponding to the free evolution Liouville super-operator is block diagonal, each block corresponding to a given coherence order

$$
\mathcal{H}_{z}^{l}=\operatorname{diag}\left(\mathcal{H}_{p p}, \mathcal{H}_{c 11}, \mathcal{H}_{c 22}, \ldots, \mathcal{H}_{c n n}\right)
$$

Physically, it means that the coherence orders do not mix within each other as well known. The unitary free evolution (without relaxation) propagator is also diagonal. The operator $\mathcal{H}_{p p}$ is identically zero since the populations are constants of the motion.

In order to proceed with relaxation, some simplifying assumptions must be made about the form of the relaxation matrix. When non-secular contributions are neglected (that is, cross-relaxation effects are neglected), the relaxation super-operator $\mathbb{R}$ has also a block diagonal structure, which leads to a complete decoupling of the relaxation of the different coherence orders

$$
\mathbb{R}=\operatorname{diag}\left(\mathbb{W}_{p p}, \mathbb{R}_{c 11}, \mathbb{R}_{c 22}, \ldots, \mathbb{R}_{c n n}\right)
$$

The submatrix $\mathbb{W}_{p p}$ drives the populations to equilibrium and gives the socalled master equation for the populations. The other square matrices $\mathbb{R}_{c k k}$ induce the relaxation of coherences to zero. If in addition the assumption of no degenerate transitions holds, then the $\mathbb{R}_{c k k}$ could be reduced to pure diagonal matrices. As concerns the formal properties needed for the calculations of the next section, we only need that $\mathcal{H}_{z}^{l}$ and $\mathbb{R}$ can be decomposed 
into a block diagonal matrix $\operatorname{diag}\left(\mathbb{A}_{p p}, \mathbb{B}_{c c}\right)$ containing no terms connecting the quantum coherences of order zero and $n \geq 1$.

We now turn to the pulse propagator. In contrast to the free evolution propagator, the pulse propagator $\mathbb{P}$ mixes all terms of the density operator. As RF pulses are generally very short compared to typical longitudinal and transversal relaxation times, we neglect the relaxation during the RF pulse (sudden approximation). With the same conventions as above, the pulse propagator is

$$
\mathbb{P}=\left(\begin{array}{c|c}
\mathbb{P}_{p p} & \mathbb{P}_{p c} \\
\hline \mathbb{P}_{c p} & \mathbb{P}_{c c}
\end{array}\right)
$$

where the different submatrices $\mathbb{P}_{p p}, \mathbb{P}_{c c}, \mathbb{P}_{c p}$ and $\mathbb{P}_{p c}$ correspond respectively to mixing 0QC (populations) within each other, 0QC and nQC, and nQC within each other. $\mathbb{P}$ is a unitary super-operator which preserves the euclidean norm of the density vector.

Also needed is the relationship between the propagators of a pulse of phase $\varphi$ and one of phase zero. The propagator of a pulse of phase $\varphi$ is related to the propagator of a pulse of phase zero by the following transformation

$$
\mathbb{P}_{\varphi}=e^{-i \varphi \mathcal{J}_{z}^{l}} \mathbb{P}_{0} e^{i \varphi \mathcal{J}_{z}^{l}}
$$

where $\mathcal{J}_{z}^{l}$ is the Liouville super-operator associated to the operator $J_{z}$. It is a general result that $0 \mathrm{QCs}$ are not affected by the pulse phase

$$
\mathbb{P}_{\varphi}\left|\rho_{p}\right\rangle=\mathbb{P}_{0}\left|\rho_{p}\right\rangle
$$




\subsection{Theory of repetitive experiments for one and two pulse sequences}

A repetitive experiment consists of the repetition of identical pulse sequences of total duration $t_{s}$ followed by a free evolution period of duration $\mathrm{T}$. The total recycle time is $T_{c}=T+t_{s} \approx T$, where we make the hypothesis that the pulse sequence duration is much smaller than the repetition period. By identical pulse sequences, we mean that all the pulses and free evolution period of the pulse sequences have the same shape, same RF power, same RF frequency, same length and same phase. Such experiments are schematized in Fig.1, for a single pulse (PRE) or Solid-Echo Repetitive Experiment (SERE). A Sequence which is a building block followed by a free evolution period of duration the recycle time $T$ is repeated many times from an initial condition $\left|\rho_{0}\right\rangle$ which may be not the equilibrium density vector. The pulses mix the coherences, while the free evolution period mix coherences of same order, and drives them back to equilibrium, which means a zero value for coherence and a non-zero value for populations, at least the Zeeman contribution. When the recycle time is much longer than the largest relaxation time, the density matrix before each sequence is the equilibrium value. On the other hand, when the recycle time has not allowed the system to relax back to equilibrium during the recycle time, a competition arise between the pulse and the free evolution periods that leads to a steady-state due to a dynamical equilibrium.

Let the propagator of the free evolution period of duration $\tau_{\alpha}$ be $\mathbb{E}_{\alpha}=\mathbb{E}_{\tau_{\alpha}}$.

\subsubsection{Steady-state density vector for a one pulse sequence, and signal}

For a single pulse, the density vector $\left|\rho_{n}\right\rangle$ just before the application of the $(n+1)^{t h}$ pulse sequence (Fig.1.Top). satisfies the iteration equation 

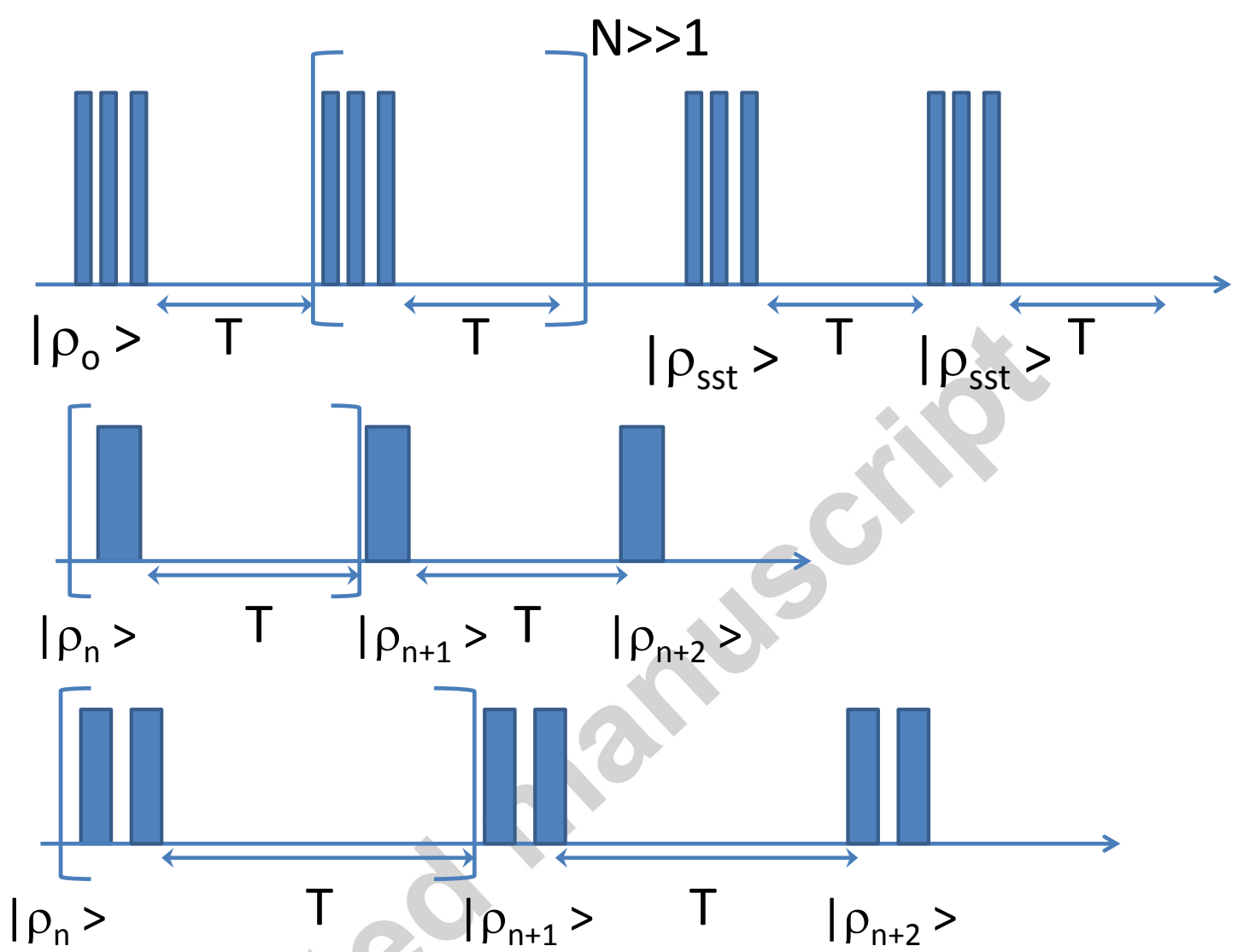

Figure 1: Repetitive experiments sequences.(Top) General Pulse sequence ;(Middle) A single pulse ;(Bottom) A two-pulse sequence. The important fact is that the steady-state is independent of the initial conditions. At each iteration, the density vector before the sequence relaxed to the equilibrium density vector $\left|\rho_{e q}\right\rangle$ only when the recycle time is long enough, typically 3-5 times the largest relaxation time. Otherwise, a steady-state is established.

$$
\left|\rho_{n}\right\rangle=\mathbb{E} \mathbb{P}\left|\rho_{n-1}\right\rangle+(\mathbb{I}-\mathbb{E})\left|\rho_{e q}\right\rangle
$$

where the free evolution (including relaxation) of duration $\mathrm{T}$ is represented by the super-operator $\mathbb{E}_{T}=\mathbb{E}$. 
If the existence of the steady-state is assumed, Eq.[16] can be directly computed by equating the steady-state density vector before a pulse and the density vector at the end of the recycle time using Eq.[14]

$$
\left|\rho_{s s t}\right\rangle=\mathbb{E P}\left|\rho_{s s t}\right\rangle+(\mathbb{I}-\mathbb{E})\left|\rho_{e q}\right\rangle
$$

This equation has the form characteristic of a feedback control system.

$$
\left|\rho_{s s t}\right\rangle=(\mathbb{I}-\mathbb{E} \mathbb{P})^{-1}(\mathbb{I}-\mathbb{E})\left|\rho_{e q}\right\rangle
$$

Equation 16 is the matrix generalization for a general spin $I \geq 1 / 2$ and a general pulse sequence of the Ernst-Anderson equation. We stress that this limit is independent of the initial conditions. Such a result was already derived in [8].

The steady-state signal recorded just after the last pulse of the sequence is calculated by noting that Eq.14 applies by changing the free propagator for duration $T, \mathbb{E}=\mathbb{E}_{t}$ to the one of duration $t, \mathbb{E}_{t}$

$$
s(t)=\left\langleI _ { - } \left|\left[\mathbb{E}_{t} \mathbb{P}\left|\rho_{s s t}\right\rangle+\left(\mathbb{I}-\mathbb{E}_{t}\right)\left|\rho_{e q}\right\rangle\right]\right.\right.
$$

with $\left|I_{-}\right\rangle$the vector associated to the operator $I_{-},\langle A \mid B\rangle$ the Euclidean scalar product between vectors $|A\rangle$ and $|B\rangle$.

\subsubsection{Steady-state density vector for a two pulse sequence, and signal}

For a two pulse sequence $\left(P_{1}\right)-\tau_{2}-\left(P_{2}\right)-T-$, the iteration equation becomes

$$
\left|\rho_{n}\right\rangle=\mathbb{E} \mathbb{P}\left|\rho_{n-1}\right\rangle+\left(\mathbb{I}-\mathbb{E}+\mathbb{E} \mathbb{P}_{2}\left(\mathbb{I}-\mathbb{E}_{\tau_{2}}\right)\right)\left|\rho_{e q}\right\rangle
$$

with $\mathbb{P}=\mathbb{P}_{2} \mathbb{E}_{\tau_{2}} \mathbb{P}_{1}$. 
Again, assuming a steady-state regime leads to

$$
\left|\rho_{s s t}\right\rangle=\left(\mathbb{I}-\mathbb{E P}_{2} \mathbb{E}_{\tau_{2}} \mathbb{P}_{1}\right)^{-1}\left(\mathbb{I}-\mathbb{E}+\mathbb{E P}_{2}\left(\mathbb{I}-\mathbb{E}_{\tau_{2}}\right)\right)\left|\rho_{e q}\right\rangle
$$

The corresponding steady-state signal is

$$
s(t)=\left\langleI _ { - } \left|\left[\mathbb{E}_{t}\left(\mathbb{P}_{2} \mathbb{E}_{\tau_{2}} \mathbb{P}_{1}\right)\left|\rho_{s s t}\right\rangle+\left(\mathbb{I}-\mathbb{E}_{t}+\mathbb{E}_{t} \mathbb{P}_{2}\left(\mathbb{I}-\mathbb{E}_{\tau_{2}}\right)\right)\left|\rho_{\text {eq }}\right\rangle\right.\right.\right.
$$

The formal structure of the steady-states of Eqs.16,19 are the same : $\left|\rho_{s s t}\right\rangle=(\mathbb{I}-\mathbb{E} \mathbb{A})^{-1}(\mathbb{I}-\mathbb{E} \mathbb{B})\left|\rho_{e q}\right\rangle . \mathbb{E}$ is the free propagator during the recycle time $T$, and it goes to zero when $T \mapsto \infty$, so that we recover the equilibrium density vector at recycle time much longer than the largest relaxation times. Superoperator $\mathbb{A}$ is the straightforward product of pulse and relaxation operators that describe the pulse sequence, $\mathbb{B}$ is more complicated. Importantly, the equilibrium density vector has only non zero contributions for the populations, the Zeeman contribution being much larger than the others. In the limit of very high field, we expect only the first column of $(\mathbb{I}-\mathbb{E} \mathbb{B})$ to contribute to the steady state.

In practice, numerical simulations should be performed to obtain results which take into account the effect of coherences. The residual coherence contributions are generally manifested by echoes in addition to the FID observed just after the pulse. However, further simplifications occur if it turns out that the coherences have been cancelled during the free evolution period. For instance, in the motional narrowing limit, the coherences which time constant contain a dominant adiabatic contribution relax generally much faster to zero than the populations to their equilibrium value. The quenching of the $\mathrm{nQC}$ coherences during the free evolution period may be obtained by the 
application of a strong magnetic field gradient. It does not affect the populations and 0QC [28]. Unfortunately, such methods usually do not apply to solids, but we can use the fact that coherences usually decay to zero at a rate much faster than the populations.

\subsubsection{The special case of quenched coherences}

It is usually verified in solid-state NMR that the recycle time can be set to verify at a time the two conditions $T_{c} \ll T \ll T_{p}$, where $T_{c}$ are typical coherences relaxation times and $T_{p}$ typical population (spin-lattice) relaxation times. Therefore, it makes sense to consider that coherences have either decayed during the recycle time, or that their weight compared to populations can be neglected. In that case, equations 16 or 19 can be greatly simplified.

The mathematical expression of the total decay to zero of the coherences is that the free evolution propagator becomes $\mathbb{E}=\operatorname{diag}\left(e^{\mathbb{W} T}, \mathbb{O}_{c c}\right)$.

For instance when a one pulse sequence is considered, the equation of the steady-state Eq.15 can be transformed to an equation only spanned by the subspace of the populations. As a matter of fact, using Eq.11, we have

$$
\begin{aligned}
\mathbb{E} \mathbb{P} & =\left(\begin{array}{c|c}
e^{\mathbb{W} T} & \mathbb{O}_{p c} \\
\hline \mathbb{O}_{c p} & \mathbb{O}_{c c}
\end{array}\right)\left(\begin{array}{c|c}
\mathbb{P}_{p p} & \mathbb{P}_{p c} \\
\hline \mathbb{P}_{c p} & \mathbb{P}_{c c}
\end{array}\right) \\
& =\left(\begin{array}{c|c}
e^{\mathbb{W} T} \mathbb{P}_{p p} & \mathbb{O}_{p c} \\
\hline \mathbb{O}_{c p} & \mathbb{O}_{c c}
\end{array}\right)
\end{aligned}
$$

Thus, only the population subspace is to be considered, leading to

$$
\left|\rho_{s s t, p p}\right\rangle=e^{\mathbb{W} T} \mathbb{P}_{p p}\left|\rho_{s s t}\right\rangle+\left(\mathbb{I}_{p p}-e^{\mathbb{W} T}\right)\left|\rho_{e q}\right\rangle
$$

and the steady-state density vector obeys 


$$
\left|\rho_{s s t, p p}\right\rangle=\left(\mathbb{I}_{p p}-e^{\mathbb{W} T} \mathbb{P}_{p p}\right)^{-1}\left(\mathbb{I}_{p p}-e^{\mathbb{W} T}\left|\rho_{e q, p}\right\rangle\right.
$$

As expected, only the population terms survive in this approximation. The resulting density vector is only a function of the submatrix $\mathbb{P}_{p p}$ of the pulse propagator which mixes the populations with each others.

For completeness, we also give the corresponding equation for a 2-pulse sequence :

$$
\left|\rho_{s s t, p p}\right\rangle=\left(\mathbb{I}_{p p}-e^{\mathbb{W} T} \mathbb{P}_{p p}\right)^{-1}\left(\mathbb{I}_{p p}-e^{\mathbb{W} T}\left(\mathbb{I}_{p p}-\mathbb{A}_{p p}\right)\right)\left|\rho_{e q, p}\right\rangle
$$

where $\mathbb{P}=\mathbb{P}_{2} \mathbb{E}_{\tau_{2}} \mathbb{P}_{1}$ and $\mathbb{A}_{p p}=\mathbb{P}_{2}\left(\mathbb{I}-\mathbb{E}_{\tau_{2}}\right)$.

The question of phase cycling has not been addressed yet. As a matter of fact, the procedure of calculation applied in the previous section is only valid when all the pulses are strictly identical. In general, the study of the steady-state of the cyclic repetition of a train of $\mathrm{n}$ different pulses is a priori more complicated in the sense that the periodicity of the steady-state regime should have the periodicity of the generating sequence. However, the problem is greatly simplified when the contribution of the coherences is neglected and when the pulses only differ by their phases, because their propagators are simply related by Eq.[12]. So, in the present context where the coherences have decayed in time $\mathrm{T}$, the population part of the density vector before the $(n+1)^{t h}$ pulse of phase $\varphi_{n+1}$ is completely determined by its population components and obeys the following recursion relation

$$
\left|\rho_{n+1, p}\right\rangle=e^{\mathbb{W} T} \mathbb{P}_{\varphi_{n+1}, p p}\left|\rho_{n, p}\right\rangle+\left(\mathbb{I}_{p}-e^{\mathbb{W} T}\right)\left|\rho_{e q, p}\right\rangle
$$


But the population part $\mathbb{P}_{\varphi_{n+1}, p p}$ of the pulse propagator $\mathbb{P}_{\varphi_{n+1}}$ is independent of the pulse phase as indicated by Eq.[13]. Thus, by following the same induction procedure as in the beginning of the section, we arrive again at Eq.[24]. It shows that the steady-state populations do not depend on the pulse phase. But, importantly, the signal recorded after each pulse is still a function of the pulse phase. Therefore, whenever the coherences have completely decayed or have been quenched during the recycle delay, a phase cycling procedure (such as CYCLOPS for instance) will be efficient on the accumulated signal. Indeed, phase cycling was used, and shown efficient, in our experiments.

\section{Application to spins $I=1$}

The theory presented above can be readily developed for spins $\mathrm{I}=1$. The formalism is greatly simplified by the fact that the relaxation of the Zeeman and Quadrupolar order are purely exponential in a first approximation.

\subsection{Liouville space for $I=1$}

The equations of motion of an isolated spin $\mathrm{I}=1$ can be found for different basis (spherical irreducible bases, single transition basis operator,...) in $[29,30,31,32]$ for example. Since numerical simulation are more efficient when performed with real matrices, we choose a Hermitian basis which can be formed by a linear combination of the spherical irreducible basis. Disregarding the identity matrix, the eight dimensional Liouville space of an isolated spin $\mathrm{I}=1$ is spanned by the orthonormal Hermitian basis

$$
\{\overbrace{B_{z}, B_{z^{2}}}^{0 Q C} \overbrace{B_{x}, B_{y}, B_{x z}, B_{y z}}^{1 Q C}, \overbrace{B_{x y}, B_{x^{2}-y^{2}}}^{2 Q C}\}
$$


These operators represent Zeeman and Quadrupolar order, four single coherence operators and two double quantum coherence operators respectively. Their explicit expressions as a function of the spin operators are

$$
\begin{gathered}
B_{i}=\frac{1}{\sqrt{2}} I_{i} ; B_{i j}=\frac{1}{\sqrt{2}}\left\{I_{i}, I_{j}\right\} \\
B_{z^{2}}=\frac{1}{\sqrt{6}}\left(3 I_{z}^{2}-2 \mathbb{I}_{3}\right) ; B_{x^{2}-y^{2}}=\frac{1}{\sqrt{2}}\left(I_{x}^{2}-I_{y}^{2}\right)
\end{gathered}
$$

where $i \neq j, i=x, y, z$, and $\{\mathrm{A}, \mathrm{B}\}=\mathrm{AB}+\mathrm{BA}$ is the anticommutator of $\mathrm{A}$ and $\mathrm{B}$, and $\mathbb{I}_{n}$ the $n \times n$ identity matrix.

The first order high field truncated free evolution Hamiltonian in the rotating frame, neglecting chemical shift effects, is

$$
H_{z}=\frac{\omega_{q}}{3}\left(3 I_{z}^{2}-2 \mathbb{I}_{3}\right)
$$

This interaction gives the typical doublet at $\pm \omega_{q}$.

The radiofrequency Hamiltonian for an irradiation at the center of the doublet is

$$
H_{r f}=\omega_{1}\left(\cos \varphi I_{x}+\sin \varphi I_{y}\right)+\frac{\omega_{q}}{3}\left(3 I_{z}^{2}-2 \mathbb{I}_{3}\right)
$$

The ratio $R=\frac{\omega_{q}}{2 \omega_{1}}$ is a measure of the hardness of the pulse, $R \ll 1$ meaning a hard pulse.

The equilibrium density vector in this basis is chosen as

$$
\left|\rho_{e q, p}\right\rangle=\left(\begin{array}{c}
\rho_{e q, z} \\
\rho_{e q, z^{2}}
\end{array}\right)=\left(\begin{array}{c}
1 \\
\frac{\omega_{q}}{\omega_{l} \sqrt{3}}
\end{array}\right)
$$

In general, the equilibrium quadrupolar order component can be neglected in high field $\rho_{e q, z^{2}} \ll \rho_{e q, z}$ (typically in a $7 \mathrm{~T}$ magnetic field, $\omega_{q} \approx 2 \pi \times 200$ $\mathrm{kHz}$ gives $\left.\frac{\omega_{q}}{\omega_{l}} \approx 4 \times 10^{-3}\right)$. 
The quadrupolar relaxation behaviour is described by five relaxation times which depend on three spectral densities. Let $T_{1 z}$ and $T_{1 q}$ be the spin-lattice relaxation time of the Zeeman and Quadrupolar order respectively, $T_{2 a}$ and $T_{2 b}$ the relaxation times of the $1 \mathrm{QC}\left(B_{x}, B_{y}\right)$ and $\left(B_{x z}, B_{y z}\right)$ respectively, and $T_{2 d q}$ the relaxation time of the 2QC. Explicit expressions in terms of spectral densities are given in [29, 30, 31]. In most case of interest where the doublet is well defined (no slow motions, $J_{1}\left(\omega_{l}\right) \ll \omega_{q}$ ), it can be shown that the relaxation of all the $1 \mathrm{QC}$ is adequately described by only one transversal relaxation time, called $T_{2}$, with $\frac{1}{T_{2}}=\frac{1}{2}\left(\frac{1}{T_{2 a}}+\frac{1}{T_{2 b}}\right)$. It is equivalent to treat the $1 \mathrm{QC}$ submatrix of the relaxation matrix as a first order perturbation of the free evolution Hamiltonian. It is important to note that the relaxation rate of the double quantum coherence does not contain any adiabatic contribution if the relaxation is assumed purely quadrupolar, whereas the two transversal relaxation times related to the 1QC have an adiabatic contribution. Therefore, in the motional narrowing limit, it can only be stated that $T_{2} \ll T_{1 z}, T_{1 q}, T_{2 d q}$.

Nevertheless, the occurrence of other non interfering first-rank like relaxation mechanisms may increase the relaxation rate of the double quantum coherences if the contribution of the corresponding adiabatic term in non negligible.

Thus, the relaxation time constants are defined as (doublets are well defined, no slow motions)

$$
\{\underbrace{T_{z}, T_{z^{2}}}_{0 Q C}, \underbrace{T_{2}, T_{2}, T_{2}, T_{2}}_{1 Q C}, \underbrace{T_{2 d}, T_{2 d}}_{2 Q C}\}
$$

For completeness, examples of pulse and free evolution superoperators 
are given in Appendix A.

\subsection{Simulations and symbolic calculations}

MATLAB [33] was used for the numerical simulations, and the Symbolic Math Toolbox, including MUPAD, to verify some results calculated by hand, or to obtain some tedious analytical results (Matlab was installed on a laptop Dell Precision M6600 with 8GB of memory, and running an Intel(R) Core(TM) i7-2820QM CPU 2.3GHz). Symbolic calculations can take a few minutes. The numerical simulation were directly performed in the Liouville space using home-made functions implemented in Matlab.

\subsection{One pulse repetitive experiments}

The steady-state density vector is calculated from Eq.16. An important result comes from the fact that only the first component of $\left|\rho_{e q}\right\rangle$ is not zero, thus the product $\mathbb{E}\left|\rho_{e q, p}\right\rangle=e_{z}\left|\rho_{e q, p}\right\rangle$ with $e_{z}=\exp \left(-T / T_{z}\right)$. The resulting steady-state density vector is thus always scaled by the factor $\left(1-e_{z}\right)$, giving

$$
\left|\rho_{s s t}\right\rangle=\left(1-e_{z}\right)(\mathbb{I}-\mathbb{E} \mathbb{P})^{-1}\left|\rho_{e q}\right\rangle
$$

where the first contribution is a scalar.

\subsubsection{Soft Pulse, quenched coherences}

Since we consider solid-state NMR, we only treat the motional narrowing limit where it might be possible to set a recycle delay such that $T_{2} \ll T \ll$ $T_{z}, T_{z^{2}}, T_{2 d}$. Since the elements of the matrix $\mathbb{E}_{11}$ related to the evolution of the $1 \mathrm{QC}$ decays in a time of the order of $3 T_{2}$, we can replace $\mathbb{E}_{11}$ by a zero matrix in this approximation and calculate the resulting steady-state density 
vector. If we divide the steady-state density vector into components related to the populations, $1 \mathrm{QC}$ and 2QC, then the solution of Eq.[16] indicates that the four components related to the 1QC are identically zero, as well as the 2QC component $\rho_{\infty, x y}$. Moreover, Eq.[35] giving the Zeeman component is also valid. The components $\rho_{\infty, z^{2}}$ and $\rho_{\infty, x^{2}-y^{2}}$ are proportional and obey a linear system of two equations. Note that these components only affect the imaginary part of the signal. In cases of practical interest, these two components are of the order of $\rho_{e q, z^{2}}$ and can be neglected when the equilibrium quadrupolar order component is vanishingly small.

For hard pulses, the matrix $\mathbb{P}_{p p}$ corresponding to the population mixing is diagonal as indicated by Eq.A.1. However, in practice, it is usually difficult to achieve the conditions of a hard pulse when the quadrupolar interaction is strong. Nevertheless, it can be shown that the corresponding $\mathbb{P}_{p p}$ matrix representing a soft pulse applied at the center of the doublet remains diagonal, but the coefficients are now complicated functions of both the pulse angle and the ratio $\omega_{q} / \omega_{1}$.

Letting $\mathbb{P}_{p p}=\operatorname{diag}\left(P_{z}, P_{z^{2}}\right)$, the solution of Eq.34 is readily calculated as

$$
\begin{aligned}
\rho_{\infty, z} & =\frac{1-e_{z}}{1-P_{z} e_{z}} \rho_{e q, z} \\
\rho_{\infty, z^{2}} & =\frac{1-e_{z^{2}}}{1-P_{z^{2}} e_{z^{2}}} \rho_{e q, z^{2}}
\end{aligned}
$$

Note that we have assumed a non-zero quadrupolar order $\rho_{e q, z^{2}}$ at equilibrium, to check the relevance of this contribution. Although derived for $\mathrm{I}=1$, these relations have the same form as the relations obtained for the $I=1 / 2$ case. Obviously, this is the consequence of the fact that $\mathbb{E}_{p p}$ and $\mathbb{P}_{p p}$ are 
diagonal, so the Zeeman and Quadrupolar order are not mixed and each contribution evolves independently of the other.

The expressions of $P_{z}$ and $P_{z^{2}}$ for a rectangular soft pulse of duration $t_{p}$, along $\mathrm{x}$, are

$$
\begin{aligned}
P_{z} & =\cos (\beta R) \cos (\beta S)+\frac{R}{S} \sin (\beta R) \sin (\beta S) \\
P_{z^{2}} & =\left(1-\frac{1}{S^{2}}\right)+\frac{1}{2 S^{2}}\left[3 \cos ^{2}(\beta S)-1\right]
\end{aligned}
$$

with $\beta=\omega_{1} t_{p}, R=\frac{\omega_{q}}{2 \omega_{1}}$ and $S=\sqrt{1+R^{2}}$. It is easy to verify that Eq.37 and 38 give the same results as a hard pulse in the limit of vanishingly small quadrupolar interaction $\left(R \rightarrow 0\right.$ and $S \rightarrow 1$ ), that is $P_{z} \rightarrow \cos \beta$ and $P_{z^{2}} \rightarrow\left(3 \cos ^{2} \beta-1\right) / 2$.

Neglecting $\rho_{e q, z^{2}}$, the measured signal is

$$
s_{z}(t)=\frac{\sin (\beta S)}{S} \frac{1-e_{z}}{1-P_{z} e_{z}} \cos \left(\omega_{q} t\right) e^{-t / T_{2}} \rho_{e q, z}
$$

Eq.39 shows that the signal amplitude, at fixed recycle time and different pulse angle, varies as $\frac{\sin (\beta S)}{S}$ when $T \gg T_{z}$, while it is proportional to

$$
\text { signal } \propto \frac{\sin (\beta S)}{S} \frac{1-e_{z}}{1-P_{z} e_{z}}
$$

in the general case of a soft pulse.

A second important point comes from the fact that the quadrupolar order $\rho_{e q, z^{2}}$ follow the same formula, by replacing $T_{1}$ by $T_{q}$.

As can be seen from Eq.[36], the contribution of Quadrupolar order will become important if the denominator is of the order of $\frac{\omega_{q}}{\omega_{l}}$. In the case of hard pulses, this is only possible for very short recycle times compared 
to $T_{z^{2}}$. In practice, one can forget the quadrupolar order contribution for spectroscopy experiments because the recycle time is of the order of $T_{z}$ (see later). However, one should be careful when progressive saturation is used to measure spin lattice relaxation time because short recycle times are used. Nevertheless, in the limit of very high field where $\rho_{e q, z^{2}}$ is vanishingly small, only the Zeeman order component remains.

When coherences are neglected, and for a hard pulse $P_{z}=\cos (\beta)$, Eq. [35] becomes exactly the equation obtained for a spin $\mathrm{I}=1 / 2$, indicating that for spins $\mathrm{I}=1$, the effective relaxation time to be considered in such experiments is the Zeeman spin-lattice relaxation time. Moreover, the signal to noise analysis given by Ernst and other workers can be directly applied [2, 3, 4, 5]. In particular, the Ernst angle $\beta_{\text {opt }}$ giving the maximum signal at fixed recycle time $\mathrm{T}$ is such that

$$
\cos \left(\beta_{o p t}\right)=\exp \left(-\frac{T}{T_{z}}\right)
$$

Such a repetitive one-pulse sequence could adequately replace the inversionsaturation experiment to measure the Zeeman spin-lattice relaxation time. It should be stressed, however, that such an analysis applies to a well defined doublet because a different correction factor must be used for each different doublet.

\subsubsection{Hard pulse and coherences}

The effects of the coherences are discussed for a hard pulse in a PRE sequence, when the coherences are allowed to evolve along the repetitive experiment. For spins $1 / 2$, it is well known that coherences give spurious signals that spoil the FID. 
The signal $s(t)=s_{r}(t)+i s_{i}(t)$ following a hard pulse along $\mathrm{x}$ and assuming $\left|\rho_{e} q\right\rangle=B_{z}$ :

$$
\begin{aligned}
s_{r}(t)= & 0 \\
s_{i}(t)= & -\sin \beta \exp \left(-t / T_{2}\right) \times \\
& {\left[f_{T} \cos \left(\omega_{q} t\right)+e_{2} g_{T} \cos \left(\omega_{q}(t-T)\right)\right] \times \frac{\left(1-e_{z}\right)}{d_{T}} }
\end{aligned}
$$

The different factors $f_{T}, g_{T}$ and the denominator $d_{T}$ are given below using the notation $c=\cos \beta, e_{2}=\exp \left(-T / T_{2}\right), e_{2 d}=\exp \left(-T / T_{2 d}\right)$

$$
\begin{aligned}
f_{T}= & \left(1-e_{2 d} \cos \beta\right) \\
g_{T}= & \left(e_{2 d}-\cos \beta\right) \\
d_{T}= & \left(1-c e_{1}\right)\left(1-c e_{2 d}\right)+e_{2} D \cos \left(\omega_{q} T\right)+ \\
& \left(e_{2}\right)^{2}\left(c-e_{1}\right)\left(c-e_{2 d}\right) \\
D= & \left(1+c^{2}\right)\left(e_{z}+e_{2 d}\right)-2 c\left(1+c e_{z} e_{2 d}\right)
\end{aligned}
$$

Note that the real part of the signal is zero even when the $1 \mathrm{Q}$ coherences have not decayed, a property which is not verified for spins $I=1 / 2$ where both the real and the imaginary components of the signal are modified.

The imaginary part of the signal is composed of two contributions. The first one, proportional to $f_{T}$, is a rescaled FID. The second contribution, proportional to $g_{T}$, is an echo because it comes from the refocusing of the $1 \mathrm{Q}$ coherences. It induces some distortions of the lineshape. However, it is easy to show that the ratio of the two signals is always lower than $e_{2}$, so this contribution may be small if $e_{2} \ll 1$ and $T_{2} \ll T_{1 z}, T_{1 q}$. 


\subsection{Solid echo repetitive experiments, coherence quenched}

In the case of a single hard pulse of angle $\beta=\pi / 2$, the amplitude of the signal reduces to $1-\exp \left(-T / T_{z}\right)$ because the denominator is equal to one.

We checked that it is also valid for a solid-echo sequence. Moreover, using a pulse angle $\beta=\pi / 2-\epsilon$, we obtained that

$$
\begin{aligned}
& s_{x}=\epsilon\left(1-\exp \left(-T / T_{z}\right)\right) \cos \left(\omega_{q} \tau\right) e^{-\frac{2 \tau}{T_{2}}}+O\left(\epsilon^{3}\right) \\
& s_{y}=-\left(1-\exp \left(-T / T_{z}\right)\right) \cos \left(\omega_{q}(t-\tau)\right) e^{-\frac{2 \tau}{T_{2}}}+O\left(\epsilon^{2}\right)
\end{aligned}
$$

The real part of the signal is zero to first order $O(\epsilon)$ while the correction to the ideal behavior for the imaginary part is in second order $O\left(\epsilon^{2}\right)$. We'll see below that experimental data are in agreement with these results.

\subsection{Simulation of the build-up of the steady-state}

To illustrate the build-up of the steady-state density matrix, for one pulse and two pulse experiments, and its convergence to a steady-state value that is independent of the initial conditions, we used an hypothetical starting initial condition were all the components of the density vector are equal to one. One iteration corresponds to the application of one sequence followed by a free evolution period of length $T$ to the density vector.

The results of the simulations are shown in Fig. 2 for short ratio $T / T_{z}=0.1$ or 0.5 . Depending on the coherence, the convergence to the steady-state can be monotone, or with oscillations. All coherences decrease to an almost zero value, except the Zeeman and Quadrupolar order, even when $T / T_{2}=1$. It seems thus that we can expect to be in a regime of "quenched coherence", where only spin-lattice relaxation times modify the signal, even for recycle times as small as $T / T_{2}=1$. 
When $T / T_{z} \geq 1$, we remarked that the memory to the initial condition is lost within only 1 to 3 iterations. For smaller ratios, as shown in the figure, the memory is lost after less than 10 iterations and the steady-state is established. This is very interesting because it shows that the steady-state is reached only after a few dummy scans, let say 16 .

These simulations explain why so many experiments give a reasonable NMR spectrum, even when the recycle time is not much larger ( 3 or 5 times) than the largest relaxation time.

\section{Experimental Results on a deuterated single crystal : $C_{19} D_{40^{-}}$ urea}

To demonstrate the relevance of our model, we used a deuterated single crystal of $\mathrm{C}_{19} \mathrm{D}_{40} / \mathrm{CO}\left(\mathrm{NH}_{2}\right)_{2}$ that presents, at room temperature, three well defined quadrupolar doublets with different spin-lattice Zeeman relaxation times $[34,35,36]$. The extend of the quadrupolar interactions in such a sample gives the opportunity to test both hard and soft pulses experimental conditions.

\subsection{Sample and experimental methods}

The ${ }^{2} H$ experiments were performed at $46 \mathrm{MHz}$ on a Bruker AVANCE 300 spectrometer with a home-made probe, and a single crystals of selectively deuterated inclusion compounds $\mathrm{C}_{19} \mathrm{D}_{40} / \mathrm{CO}\left(\mathrm{NH}_{2}\right)_{2}$. The crystal was grown following conventional procedure and using fully deuterated alkane $C_{19} D_{40}$ of commercial origin (Eurisotop). The crystal c-axis was put perpendicular to the external magnetic field, to have the $C-D_{2}$ bonds in a plane that contains the magnetic field. The irradiation frequency was always chosen 

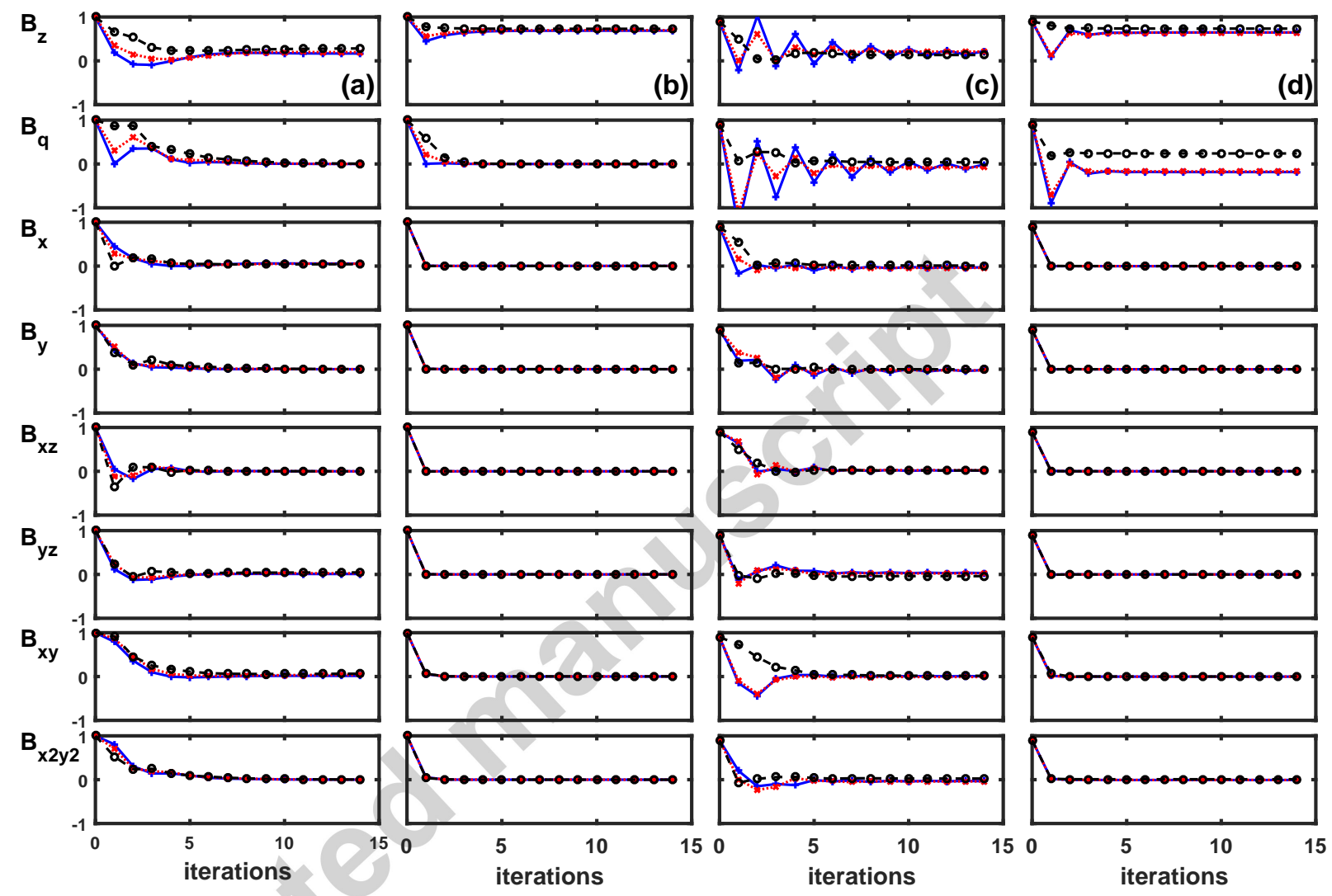

Figure 2: Build-up of the steady-state density matrix as a function of the iteration number, starting with an hypothetical initial condition where all components of the density matrix are equal to one. The parameters $R=0.2,0.5,1$ for (continuous blue + ), (dotted red line $\mathrm{x}$ ), (dashed black o) respectively. Pulse angle is $45^{\circ}$. Interpulse delay $\tau=50 \mu \mathrm{s}$. (a) PRE with $T / T_{z}=0.1 ; T / T_{q}=0.1 ; T / T_{2}=1 ; T / T_{2 d}=0.7 ;$ (b) PRE with $T / T_{z}=0.5 ; T / T_{q}=0.5 ; T / T_{2}=5$ $; T / T_{2 d}=3.3 ;$ (c) SERE with $T / T_{z}=0.1 ; T / T_{q}=0.1 ; T / T_{2}=1 ; T / T_{2 d}=0.7 ;$ (d) SERE with $T / T_{z}=0.5 ; T / T_{q}=0.5 ; T / T_{2}=5 ; T / T_{2 d}=3.3 ;$ 
at the center of the doublet, to cope with the theory developed in the first part. The Zeeman spin-lattice relaxation times of the different lines were measured with an inversion-recovery sequence. For steady-state experiments, the spectra were acquired after a number of dummy scans of 64 that ensure that the steady-state is reached. For variable pulse length experiment, the maximum pulse length (or pulse angle) was limited by the probe arcing or electrical breakdown. Single pulse and two-pulse solid-state echo were used, at two radiofrequency power $\nu_{1}=36 \mathrm{kHz}$ and $\nu_{1}=16 \mathrm{kHz}$.

\subsection{Results and discussion}

The spectra obtained from a solid-echo repetitive experiment at three different recycle times are presented in Fig.3. The experimental parameters of the three different lines are summarized in Table.1.

From these values, we note that the coherences are negligible as long as the recycle time is larger than $30-40 \mathrm{~ms}$. This simplifies greatly the interpretation of the data.

\subsubsection{One pulse repetitive experiment as a function of pulse angle}

For spins $1 / 2$, the steady-state sequence was used at fixed $\mathrm{T}$ as a function of pulse angle, either to maximize the SNR, or to estimate $T_{1}$ from the angle corresponding to the maximum signal value. As shown before, the Ernst formula is also valid for a single pulse $\mathrm{I}=1$ experiment. Figure.4 demonstrates that experimental curves are well reproduced by Eq.40, with all parameters given by experimental conditions, with no adjustable parameters. 


\begin{tabular}{|l|c|c|c|}
\hline & $C D_{3}$ & $C D_{2 \alpha}$ & $C D_{2}^{\text {bulk }}$ \\
\hline$\nu_{q}(\mathrm{kHz})$ & 7.8 & 25.4 & 30 \\
$T_{z}(\mathrm{~ms})$ & 360 & 680 & 2400 \\
\hline$\nu_{1}=16 \mathrm{khz}$ & & & \\
$R$ & 0.24 & 0.79 & 0.94 \\
$S$ & 1.03 & 1.28 & 1.37 \\
$R / S$ & 0.24 & 0.62 & 0.68 \\
\hline$\nu_{1}=36 \mathrm{khz}$ & & & \\
$R$ & 0.11 & 0.35 & 0.42 \\
$S$ & 1.01 & 1.06 & 1.08 \\
$R / S$ & 0.11 & 0.33 & 0.38 \\
\hline
\end{tabular}

Table 1: Experimental data of the three quadrupolar doublets of $C_{19} D_{40} / C O\left(\mathrm{NH}_{2}\right)_{2}$ at room temperature. The value of the parameters $R=\frac{\nu_{q}}{2 \nu_{1}}$ and $S=\sqrt{1+R^{2}}$ that are used in soft pulses are also given. For all doublets, the $T_{2}$ are smaller than 5-10ms.

\subsubsection{One pulse and solid-echo repetitive experiment as a function of recycle time}

For a given pulse sequence, the signal amplitude can be varied as a function of the interpulse delay $T$. As for spins $I=1 / 2$, the question is whether such a "progressive saturation" experiment can also be used to measure the spin-lattice Zeeman relaxation time $T_{z}$. The experimental results for both single pulse and echo sequence are shown in Fig.5.

For a PRE, the theoretical predictions from Eq.40 are in good agreement with the experimental results, as indicated by Fig.5.(Left). Remark that no variable parameters are used, since all parameters needed in the analytical 
formula were already measured. The agreement between experiment and theory is a notable affirmation of the relevance of the generalized Ernst-Anderson formula for spin $\mathrm{I}=1$. Moreover, it indicates that such a progressive saturation experiment can be used to estimate the Zeeman spin lattice relaxation time, by fitting the data with

$$
s(T)=a_{2}+a_{3} * \frac{(1-\exp (-a 1 * T))}{\left(1-P_{z} * \exp (-a 1 * T)\right)}
$$

with $a 1 \approx 1 / T_{z} . a_{2}$ and parameters $a_{3}$ represent the background and amplitude, as in a general 3-parameter fit in a classical relaxation experiment (saturation-recovery or inversion-recovery). For irradiation at the center of the doublet, $P_{z}$ can be calculated as explained above from Eq.37 and the known experimental parameters.

For the SERE, the simplified formula Eq.43 approximate well the experimental for $C D_{3}, C D_{2 \alpha}$, but not for $C D_{2}^{\text {bulk }}$, as exemplified in Fig.5.(Right). This was to be expected since Eq.43 was derived from the hypothesis of an ideal solid-echo sequence with hard pulses of angles $\pi / 2 \equiv 90^{\circ}$. In the experiment, a smaller pulse angle $\approx 64^{\circ}$ was used to cover the full spectral width, and insure that the spectrum could be phased. From the theory, we expect a signal amplitude that should contain a numerator that behaves almost like $1-a * \exp \left(-T / T_{z}\right)$, and a denominator that is dependent on pulse angle, recycle time and quadrupolar frequency and relaxation times.

Because useful analytical formula for SERE could not be obtained, a full numerical simulation of the signal intensity was performed, which results are superimposed on the data as red lines Fig.5. Again, no adjustable parameters were used. The agreement between the simulations and the experiment is now good, and confirms again the applicability of the theory to explain these 
experiments. In addition, a fit with the simplified formula would also give a good approximation of $T_{z}$.

\section{Conclusion}

In this study, the results of the Ernst-Anderson model obtained for the one-pulse repetitive experiment on an isolated spin $\mathrm{I}=1 / 2$ were generalized to the case of general pulse sequences and general spin values $I \geq 1 / 2$ by using the Liouville space formalism for high field NMR. The resulting formula have a similar analytical structure $\left|\rho_{\text {sst }}\right\rangle=(\mathbb{I}-\mathbb{B})^{-1}(\mathbb{I}-\mathbb{A})\left|\rho_{\text {eq }}\right\rangle$, but instead of scalars, $\mathbb{A}$ and $\mathbb{B}$ are super-operators depending on the pulse sequence and relaxation.

The main assumptions which made the problem tractable are the following : (1) High field and high temperature approximation ; (2) the relaxation is neglected during the pulses, but the pulses can be either hard or soft. (3) during the free evolution period, the relaxation is treated in the weak regime, such that the Redfield evolution equation for the density operator applies ; (4) the non-secular components of the relaxation operator are neglected, that is no cross relaxation occurs ; formally, we only need a decoupling between the $0 \mathrm{QC}$ and $\mathrm{nQC}$.

The fundamental expression presented in this work are Eq.[16] and [19]. The steady-state regime is independent of the initial conditions. Additional results were obtained by assuming that the coherences can be quenched during the recycle time period. If this is so, we showed that the steady-state is only a function of the populations Eq.[24] and [25]. In the simplest case of a single pulse sequence, it is independent of the pulse phase, and a phase cy- 
cling procedure like CYCLOPS to remove pulse/receiver imperfections should work.

We applied the formalism to spins $I=1$ subjected to a non zero quadrupolar interaction, and a pulse sequence composed of hard or soft pulses with irradiating frequency at the centre of the doublet. We were able to generalize the Ernst-Anderson formula for the Zeeman order and Quadrupolar order. These results on spins $\mathrm{I}=1$ may have practical use because ${ }^{2} \mathrm{H}$ deuterium and ${ }^{14} N$ NMR are powerful techniques to study molecular systems. Practically, as soon as the experimentalist is able to irreversibly quench the coherences before the application of a sequence, then the experimental accumulation time of a simple one-pulse or solid-echo sequence can be reduced by using a recycle time of the order of the Zeeman order relaxation time instead of at least five times this value. All the predictions of the theory were verified by experiments performed on deuterated nonadecane/urea single crystal.

Further work is needed to treat more general cases, like selective excitation of the central transition of half-integer spins, to know whether repetitive experiments at short recycle time can be used to obtain spectral or dynamical information, but we think that including relaxation in the formal treatment of spin dynamics in the Liouville space for the study of pulse sequences is only at its beginning for solid-state quadrupolar NMR.

\section{APPENDIX}

\section{Appendix A. Example of Spin $I=1$ superoperators}

The unitary propagator of a hard pulse of angle $\beta=\omega_{1} t_{p}$, along $\mathrm{x}$ or $\mathrm{y}$, applied at the center of the doublet can be represented by the orthogonal 
matrix

$\mathbb{P}_{x}=$

$$
\left(\begin{array}{cc|cccc|cc}
c_{\beta} & 0 & 0 & s_{\beta} & 0 & 0 & 0 & 0 \\
0 & \frac{1}{2}\left(3 c_{\beta}^{2}-1\right) & 0 & 0 & 0 & \frac{\sqrt{3}}{2} s_{2 \beta} & 0 & -\frac{\sqrt{3}}{2} s_{\beta}^{2} \\
\hline 0 & 0 & 1 & 0 & 0 & 0 & 0 & 0 \\
-s_{\beta} & 0 & 0 & c_{\beta} & 0 & 0 & 0 & 0 \\
0 & 0 & 0 & 0 & c_{\beta} & 0 & s_{\beta} & 0 \\
0 & -\frac{\sqrt{3}}{2} s_{2 \beta} & 0 & 0 & 0 & c_{2 \beta} & 0 & -\frac{1}{2} s_{2 \beta} \\
\hline 0 & 0 & 0 & 0 & -s_{\beta} & 0 & c_{\beta} & 0 \\
0 & -\frac{\sqrt{3}}{2} s_{\beta}^{2} & 0 & 0 & 0 & \frac{1}{2} s_{2 \beta} & 0 & 1-\frac{1}{2} s_{\beta}^{2}
\end{array}\right)
$$

$\mathbb{P}_{y}=$

$$
\left(\begin{array}{cc|cccc|cc}
c_{\beta} & 0 & -s_{\beta} & 0 & 0 & 0 & 0 & 0 \\
0 & \frac{1}{2}\left(3 c_{\beta}^{2}-1\right) & 0 & 0 & -\frac{\sqrt{3}}{2} s_{2 \beta} & 0 & 0 & \frac{\sqrt{3}}{2} s_{\beta}^{2} \\
\hline-s_{\beta} & 0 & c_{\beta} & 0 & 0 & 0 & 0 & 0 \\
0 & 0 & 0 & 1 & 0 & 0 & 0 & 0 \\
0 & \frac{\sqrt{3}}{2} s_{2 \beta} & 0 & 0 & c_{2 \beta} & 0 & 0 & -\frac{1}{2} s_{2 \beta} \\
0 & 0 & 0 & 0 & 0 & c_{\beta} & -s_{\beta} & 0 \\
\hline 0 & 0 & 0 & 0 & 0 & s_{\beta} & c_{\beta} & 0 \\
0 & \frac{\sqrt{3}}{2} s_{\beta}^{2} & 0 & 0 & \frac{1}{2} s_{2 \beta} & 0 & 0 & 1-\frac{1}{2} s_{\beta}^{2}
\end{array}\right)
$$

where $c_{\beta}=\cos \beta, c_{2 \beta}=\cos 2 \beta$ etc... The free propagator $\mathbb{E}_{t}$ is 


$$
\mathbb{E}_{t}=\left(\begin{array}{cc|cccc|cc}
e_{z} & 0 & 0 & 0 & 0 & 0 & 0 & 0 \\
0 & e_{z^{2}} & 0 & 0 & 0 & 0 & 0 & 0 \\
\hline 0 & 0 & c_{t} e_{2} & 0 & 0 & -s_{t} e_{2} & 0 & 0 \\
0 & 0 & 0 & c_{t} e_{2} & s_{t} e_{2} & 0 & 0 & 0 \\
0 & 0 & 0 & -s_{t} e_{2} & c_{t} e_{2} & 0 & s & 0 \\
0 & 0 & s_{t} e_{2} & 0 & 0 & c_{t} e_{2} & 0 & 0 \\
\hline 0 & 0 & 0 & 0 & 0 & 0 & e_{2 d} & 0 \\
0 & 0 & 0 & 0 & 0 & 0 & 0 & e_{2 d}
\end{array}\right)
$$

where

$e_{z}=\exp \left(-t / T_{z}\right), e_{z}^{2}=\exp \left(-t / T_{q}\right), e_{2}=\exp \left(-t / T_{2}\right), e_{2 d}=\exp \left(-t / T_{2 d}\right), c_{t}=\cos \left(\omega_{q} t\right)$ and $s_{t}=\sin \left(\omega_{q} t\right)$

Funding : This research received FEDER financial support (FEDER 34722Prin2Tan) for funding NMR spectrometers.

Acknowledgments : I would like to thank Doctor Bertrand Toudic for his support and comments, and Claire Roiland and Laurent Le Polles for fruitful discussions.

[1] H.Y. Carr, Steady-State Free Precession in Nuclear Magnetic Resonance, Phys. Rev. 112, 1693-1701 (1958).

[2] R.R. Ernst and W.A. Anderson, Application of Fourier transform spectroscopy to magnetic resonance, Rev. Sci. Instrum. 37, 93-102 (1966).

[3] R. Freeman and H.D.W. Hill, Fourier transform study of NMR spin lattice relaxation by "progressive saturation", J. Chem. Phys. 54, 33673377 (1971) 
[4] R. Freeman and H.D.W. Hill, Phase and intensity anomalies in Fourier transform NMR, J. Magn. Reson. 4, 366-383 (1971).

[5] D.E. Jones and H. Sternlicht, Fourier transform nuclear magnetic resonance I. repetitive pulses, J. Magn. Reson. 6, 167-182 (1972).

[6] R.G.S. Spencer, J.A. Ferreti, G.H. Weiss, NMR saturation factors in the presence of chemical exchange, J. Magn. Reson. 84, 223-235 (1989).

[7] R.G.S. Spencer and K.W. Fishbein, Measurement of spin-lattice relaxation times and concentrations in systems with chemical exchange using the one-pulse sequence : breakdown of the Ernst Model for partial saturation in nuclear magnetic resonance spectroscopy, J. Magn. Reson. 142, 120-135 (2000).

[8] C.K. Anand, A. D. Bain, Z. Nie, Simulation of steady-state $\{$ NMR $\}$ of coupled systems using Liouville space and computer algebra methods Journal of Magnetic Resonance 189, 200-208 (2007).

[9] V. F. Mitrović, E. E. Sigmund, W. P. Halperin, Progressive saturation NMR relaxation Phys. Rev. B 64, 024520 (2001).

[10] N. Chandrakumar, The Basic Physics of Spin-1 Systems, in Spin-1 NMR ,Springer Berlin Heidelberg, 3-26 (1996).

[11] N. Chandrakumar, NMR of Spin-1 Systems in the Solid State, in Spin-1 NMR ,Springer Berlin Heidelberg, 59-109 (1996).

[12] K. Schmidt-Rohr, and H.W. Spiess, Multidimensional Solid-state NMR and Polymers, Academic Press (1994). 
[13] E. Dib, T. Mineva, B. Alonso, Chapter Three - Recent Advances in $14 \mathrm{~N}$ Solid-State $\{\mathrm{NMR}\}$, in Annual Reports on $\{N M R\}$ Spectroscopy ,Academic Press, Ed.G.A. Webb, Vol.87, pp 175-235 (2016).

[14] P. Speier, G. Prigl, H. Zimmermann, U. Haeberlen, E. Zaborowski, S.Vega, The site and dynamics of p-xylene guest molecule in Dianin's inclusion compound; a Deuteron NMR study Appl. Magn. Reson,9, 81$102(2000)$.

[15] R.R. Vold and R.L. Vold, Deuterium Relaxation in Molecular Solids, in Adv. in Magn. and Opt. Res. , Academic Press, New-York, 16, 85-171 (1991).

[16] J.H. Davis, The description of membrane lipid conformation, order and dynamics by ${ }^{2}-\mathrm{H}$ NMR Biochimica et Biophysica Acta,737, 117-171 (1983).

[17] G. Slosarek, A. Heuer, H. Zimmermann, U. Haeberlen, A study of the paraelectric-ferroelectric phase transition of triglycine sulphate by deuteron nuclear magnetic resonance and relaxation, J. Phys.: Condens. Matter, 1, 5931-5954 (1989).

[18] T.M. Alam, G.P. Drobny, Solid-State NMR studies of DNA structure and dynamics, Chem. Rev. , 91, 1545-1590 (1991).

[19] S. Benz, U. Haeberlen, Jump motion of Deuterons along hydrogen bonds in $\mathrm{KDCO}_{3}$, J. Magn. Res., 66, 125-134 (1986).

[20] A.G. Redfield, The theory of relaxation processes, in Adv. in Magn. Reson. 1, ed. by J. S. Waugh, Academic Press, New York, 1-32 (1965). 
[21] R.R. Ernst, G. Bodenhausen, and A. Wokaun, "Principles of Nuclear Magnetic Resonance in One and Two Dimensions", Clarenton Press, Oxford (1994).

[22] C.P. Slichter, "Principles of Magnetic Resonance", 3rd ed. SpringerVerlag, Berlin/New York (1990).

[23] R.A. Hoffman, Line shapes in High-Resolution NMR in Adv. in Magn. Reson. 4, ed. by J. S. Waugh, Academic Press, New York, 88-200 (1970).

[24] U. Fano, "Liouville Representation of Quantum Mechanics with Application to Relaxation Processes", In : Caianiello E.R., editor, Lectures on The Many-Body Problem, New-York : Academic Press; 217-239 (1964).

[25] A.D. Bain, The Superspin Formalism for Pulse NMR, Prog. NMR Spectros.,20, 295-315 (1988).

[26] R. Ghose, Average Liouvillian Theory in Nuclear Magnetic Resonance Principles, Properties, and Applications, Concepts. Magn. Res.,12, 152$172(2000)$.

[27] A. D. Bain, B. Berno, Liouvillians in NMR: The direct method revisited Progress in Nuclear Magnetic Resonance Spectroscopy 59, 223-244 (2011).

[28] Wokaun and R.R. Ernst, Selective detection of multiple quantum transitions in NMR by two-dimensional spectroscopy, Chem. Phys. Lett. 52, 407-412 (1977). 
[29] J.P. Jacobsen, H. K. Bildsoe, K. Shaumburg, Application of density matrix formalism in NMR spectroscopy. II. The One-spin-1 Case in anisotropic phase. J. Magn. Reson. 23, 153-164 (1976).

[30] K.R. Jeffrey, Nuclear magnetic relaxation in a spin 1 system, Bull. Magn. Reson. 3, 69-82 (1981).

[31] S.B. Ahmad, K.J. Packer, J.M. Ramsden, The dynamics of water in heterogeneous systems. II. Nuclear magnetic relaxation of the protons and deuterons of water molecules in a system with identically oriented planar interfaces., Mol. Phys. 33, 857-874 (1977).

[32] C. Schmidt, B. Blumich and H.W. Spiess, Deuteron two-dimensional exchange NMR in solids, J. Magn. Reson. 79, 269-290 (1988).

[33] MATLAB and Symbolic Math Toolbox(Version 7.0) R2016a, The MathWorks, Inc., Natick, Massachusetts, United States.

[34] Le Lann, H. and Odin, C. and Toudic, B. and Ameline, J. C. and Gallier, J. and Guillaume, F. and Breczewski, T.,Single-crystal deuterium NMR study of the symmetry breaking in an incommensurate organic inclusion compound, Phys. Rev. B 62, 5442-5451 (2000).

[35] El Baghdadi, A. and Dufourc, E. J. and Guillaume, F., hain End Dynamical and Conformational Properties of n-Nonadecane Molecules in Urea Inclusion Compounds: A Study by Deuterium NMR Spectroscopy, The Journal of Physical Chemistry 100, 1746-1752 (1996).

[36] M.S.Greenfield,R.L.Vold, R.R.Vold , Deuterium spin relaxation and 
guest motion in the n-nonadecane/urea clathrate, Molecular Physics 66, 269-298 (1989). 


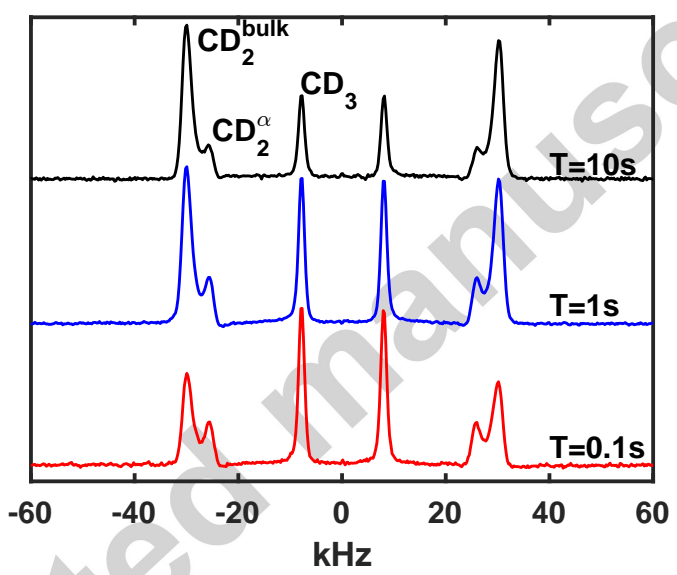

Figure 3: Solid-Echo repetitive experiment at three different recycle times. The experimental parameters are : pulse angle $\beta=64^{\circ}$, interpulse delay $\tau=50 \mu \mathrm{s}, \nu_{1}=36 \mathrm{kHz}$. Spectra obtained by FFT of half of the echo (taken from the top of the echo). 

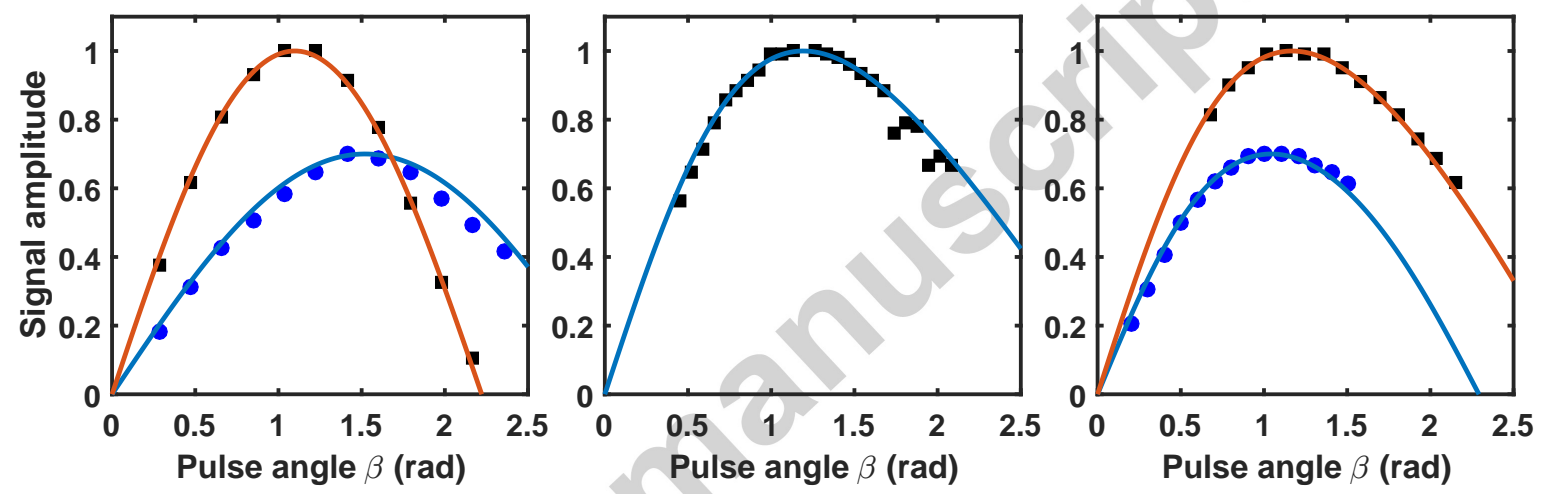

Figure 4: One pulse experiment : signal amplitude as a function of pulse angle. Markers are experimental data while continuous lines where obtained from Eq.40 with no fitting parameters. (Left) Fully relaxed with recycle time $\mathrm{T}=5 \mathrm{~s}$ for $C D_{3}(\bullet)$ and $C D_{2}^{\text {bulk }}$ at $\nu_{1}=36 \mathrm{kHz}$.(Center) $C D_{3}$ at $T=T_{z}$ and $\nu_{1}=36 \mathrm{kHz}$. (Right) $C D_{2}^{\text {bulk }}$ and $T=T_{z}$ for two radiofrequency strengths : (汭 $\nu_{1}=36 \mathrm{kHz}$ and $(\boldsymbol{\bullet}) \nu_{1}=16 \mathrm{kHz}$. In figure (Left) and (Right), the maximum amplitude of the two signals were arbitrarily scaled to 1 and 0.7 for sake of clarity. 

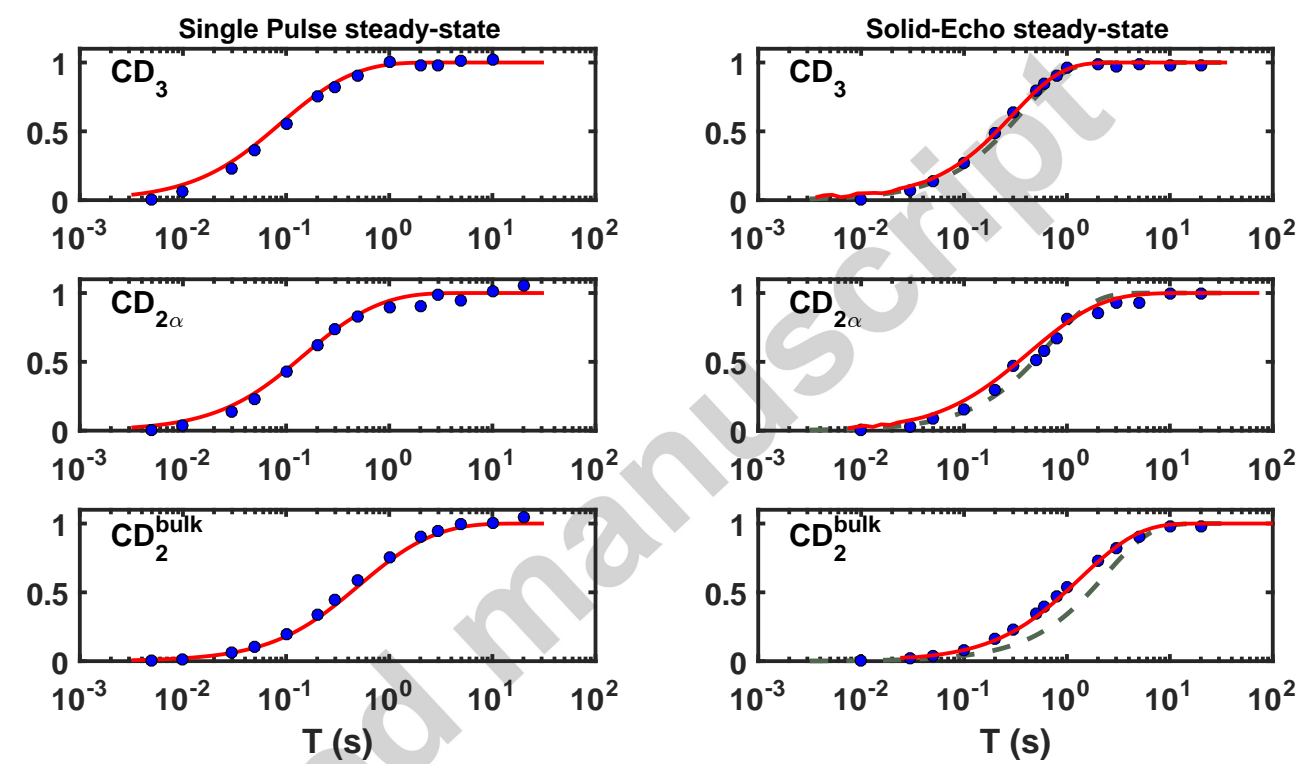

Figure 5: Signal amplitude as a function of recycle time T. (Left) Single Pulse with $\mathrm{P} 1=7 \mathrm{u}$ and $\nu_{1}=16 \mathrm{kHz}$ (points). The red continuous lines are calculated from Eq.40 using the data of table. 1 and the values of $P_{z}=0.779,0.799,0.807$ for $C D_{3}, C D_{2 \alpha}$ and $C D_{2}^{\text {bulk }}$ respectively. (Right) Solid-Echo sequence $\left(P_{x}-\tau-P_{y}\right.$ using a 8 phases cycling procedure, with $P_{x}=P_{y}=11 \mu \mathrm{s}, \nu_{1}=16 \mathrm{kHz}$, and interpulse delay $\tau=50 \mu \mathrm{s}$. The gray dashed lines are obtained from $E(T)=\left(1-e^{-T / T_{z}}\right)$. The red continuous lines come from the numerical simulation of steady-state solid-echo sequence. 